\title{
Modeling of the concrete-bentonite interface in nuclear waste disposal systems: from pore-scale to continuum scale
}

\author{
HANG DENG ${ }^{1}$, LIANGE ZHENG $^{2}$, YUTONG MU ${ }^{3}$ AND PEI \\ $\mathrm{LI}^{1}$ \\ ${ }^{1}$ Lawrence Berkeley National Laboratory \\ ${ }^{2}$ Lawrence Berkeley National Lab \\ ${ }^{3} \mathrm{Xi}$ 'an Jiaotong University \\ Presenting Author: hangdeng@lbl.gov
}

Long-term storage of nuclear waste in geological formations is critical for the use of nuclear energy. In the multi-barrier system for nuclear waste disposal, interfacial areas such as canistersbentonite, concrete-bentonite, and concrete liners - host rocks, are particularly susceptible to alteration due to distinct chemical and physical properties of the materials. The resulting chemical reactions within $\mathrm{mm}-\mathrm{cm}$ of the interfaces can significantly change the transport properties of the materials, and thus have a profound impact on the migration of the radionuclides.

To describe more accurately the drastic changes in the minerals phase in the vicinity of the interface and the resulting changes in pore structure and permeability, pore-scale modeling is needed. In this study, we use both the continuum reactive transport model and the Lattice Boltzmann Method (LBM) to investigate the reactive transport processes at the concretebentonite interfaces. Both models simulate the coupled physical processes including the single-phase fluid flow, ion transport, and the dissolution and precipitation of minerals. After the reaction paths in the continuum scale model are confirmed by data from a long-term field, there were implemented in the LBM model to study the microstructural change of concrete and bentonite around the interfacial area. Model results show that the concrete side is characterized by the dissolution of portlandite, ettringite, and calcite, the formation of Calcium-AluminiumSilicate-Hydrates, and secondary ettringite where bentonite is featured with the dissolution of primary accessory minerals such as gypsum and precipitation of secondary phases such calcite, brucite and hydrotalcite. An increase in porosity and surface area has been observed on both sides of the interface. While LBM seems to be a promising method to study chemical alteration and the resulting change in transport properties, but the required extremely high spatial resolution and long simulation time make it very challenging. 